\title{
Modulation of the cortical silent period elicited by single- and paired-pulse transcranial magnetic stimulation
}

Sho Kojima ${ }^{1,2^{*}}$, Hideaki Onishi ${ }^{2}$, Kazuhiro Sugawara ${ }^{2}$, Hikari Kirimoto ${ }^{2}$, Makoto Suzuki ${ }^{2}$ and Hiroyuki Tamaki ${ }^{2}$

\begin{abstract}
Background: The cortical silent period (CSP) elicited by transcranial magnetic stimulation (TMS) is affected by changes in TMS intensity. Some studies have shown that CSP is shortened or prolonged by short-interval intracortical inhibition (SICI) and intracortical facilitation (ICF), Those studies, however, used different TMS intensities to adjust the amplitude of the motor evoked potential (MEP). Therefore, it is unclear whether changes in CSP duration are induced by changes in TMS intensities or by SICI and ICF. The purpose of this study was to confirm the effects of muscle contractions and stimulus intensities on MEP amplitude and the duration of CSP induced by single-pulse TMS and to clarify the effects of SICl and ICF on CSP duration.

MEP evoked by TMS was detected from the right first dorsal interosseous muscle in 15 healthy subjects. First, MEP and CSP were induced by single-pulse TMS with an intensity of 100\% active motor threshold (AMT) at four muscle contraction levels $[10 \%, 30 \%, 50 \%$, and 70\% electromyogram (EMG)]. Next, MEP and CSP were induced by seven TMS intensities $(100 \%, 110 \%, 120 \%, 130 \%, 140 \%, 150 \%$, and 160\% AMT) during muscle contraction of 10\% EMG. Finally, $\mathrm{SICl}$ and ICF were recorded the four muscle contraction levels (0\%, 10\%, 30\%, and 50\% EMG).

Results: MEP amplitudes increased with increases in muscle contraction and stimulus intensity. However, CSP duration did not differ at different muscle contraction levels and was prolonged with increases in stimulus intensity. CSP was shortened with SICl compared with CSP induced by single-pulse TMS and with ICF at all muscle contraction levels, whereas CSP duration was not significantly changed with ICF.

Conclusions: We confirmed that CSP duration is affected by TMS intensity but not by the muscle contraction level. This study demonstrated that CSP is shortened with $\mathrm{SICl}$, but it is not altered with ICF. These results indicate that after $\mathrm{SICl}$, CSP duration is affected by the activity of inhibitory intermediate neurons that are activated by the conditioning $\mathrm{SICl}$ stimulus.
\end{abstract}

Keywords: Transcranial magnetic stimulation, Motor evoked potential, Cortical silent period, Short-interval intracortical inhibition, Intracortical facilitation

\section{Background}

Motor evoked potential (MEP) is recorded from peripheral muscles after stimulation of the primary motor cortex by transcranial magnetic stimulation (TMS). TMS produces MEP followed by a period of electromyogram (EMG) silence during voluntary muscle contraction. This period

\footnotetext{
* Correspondence: hpm11003@nuhw.ac.jp

${ }^{1}$ Graduate school of Health and Welfare, Niigata University of Health and Welfare, Nigata, Japan

${ }^{2}$ Institute for Human Movement and Medical Sciences, Niigata University of Health and Welfare, 1398 Shimami-cho, Kita-ku, Niigata City, Niigata 950-3198, Japan
}

\section{Biomed Central

(c) 2013 Kojima et al.; licensee BioMed Central Ltd. This is an Open Access article distributed under the terms of the Creative Commons Attribution License (http://creativecommons.org/licenses/by/2.0), which permits unrestricted use, distribution, and reproduction in any medium, provided the original work is properly cited. of silence is known as the cortical silent period (CSP). While spinal mechanisms may be active in the early part (approximately $50 \mathrm{~ms}$ ) of CSP, the cortical origin of at least the later part (approximately $100 \mathrm{~ms}$ ) of CSP has been proven in several previous studies [1-5]. MEP amplitude fluctuates with TMS intensity, muscle contraction [6-8], and the difficulty of the task [9]. Several studies have investigated the relationship between MEP amplitude and CSP duration; however, this relationship remains unclear. For example, Wu et al. [10] reported that a large MEP amplitude induces CSP for a long duration, whereas Gilio et al. 
[11] found that CSP duration was not related to the MEP amplitude.

Recently, paired-pulse TMS has become a useful tool for testing cortical inhibition or facilitation of the human motor cortex. When a subthreshold conditioning pulse (S1) and a suprathreshold test pulse (S2) were applied to the motor cortex through the same coil, MEP evoked by the test pulse was inhibited at interstimulus intervals (ISI) between 1 and $5 \mathrm{~ms}$ [12]. This phenomenon is known as short-interval intracortical inhibition (SICI) [12-14]. The mechanism of SICI was reported to result from synaptic interaction occurring within M1, and it appeared to be mediated at the cortical level $[12,15]$. Therefore, a subthreshold S1 suppressed the size of both the descending spinal cord volleys and MEP induced by a suprathreshold S2 [16]. In pharmacological research, the mechanism of SICI has been explored in more detail, and the involvement of gamma-aminobutyric acid (GABAA) has been suggested [14,17-20]. In contrast, MEP is facilitated at ISI greater than $10 \mathrm{~ms}$, which is known as intracortical facilitation (ICF) [12-14]. Because the intensity of the conditioning stimulus was too weak for enhancement of any effects on spinal $\mathrm{H}$ reflexes, it was considered that ICF occurred within the cerebral cortex $[12,14]$. However, Di Lazzaro et al. [21] suggested the possibility that the conditioning stimulus reflects the excitability of spinal motoneurons. Although there is evidence for the origin of SICI, there is less direct information on the origin of ICF.

There have been some studies concerning CSP duration after SICI or ICF during muscle contraction
[22-24], whereas many studies of SICI or ICF have been conducted under resting conditions [15,25,26]. MEP amplitude induced by paired-pulse TMS was reported to change during muscle contraction at $5 \%-50 \%$ of maximum voluntary contraction (MVC) $[19,25]$. Some studies showed that CSP was shortened or prolonged by paired-pulse TMS [22-24]; however, these studies used different intensities of paired-pulse TMS to adjust the MEP amplitude. Therefore, it is unclear whether changes in CSP duration are induced by the change in magnetic stimulation intensities or by SICI or ICF.

The purpose of this study was to confirm the effects of muscle contractions and stimulus intensities on MEP amplitude and the duration of CSP induced by singlepulse TMS and to clarify the effects of SICI and ICF on CSP duration.

\section{Results}

The $1 \mathrm{mV}$ TMS intensity and active motor threshold (AMT) were $56.5 \pm 7.5 \%$ and $36.0 \pm 4.7 \%$ [mean \pm standard deviation (SD)], respectively, of the maximum stimulator output. Figure 1 shows representative waveforms of MEP during muscle contraction of 30\% EMG. We were able to clearly observe CSP for all muscle contraction levels and all TMS intensities.

\section{Effects of muscle contraction on MEP amplitude and CSP duration (experiment 1)}

Results of one-way analysis of variance (ANOVA) showed that MEP amplitude changed significantly with increased muscle contraction $\left(\mathrm{F}_{(3,30)}=45.758, \mathrm{p}<0.01\right.$; Figure 2a).

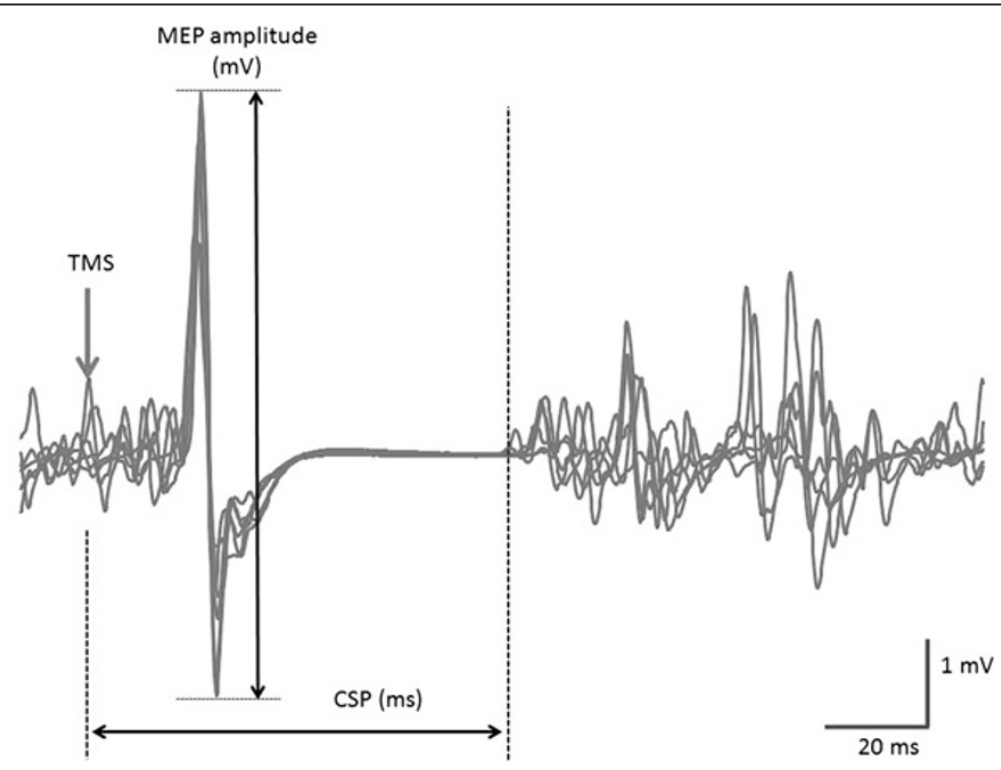

Figure 1 Representative waveforms of MEP induced by TMS during sustained 30\% EMG. MEP amplitude was calculated by averaging peak-to-peak amplitudes. CSP duration was defined as the time from TMS onset to the time of reappearance of an EMG amplitude that was 3-fold the standard deviation of the background EMG noise at rest. 

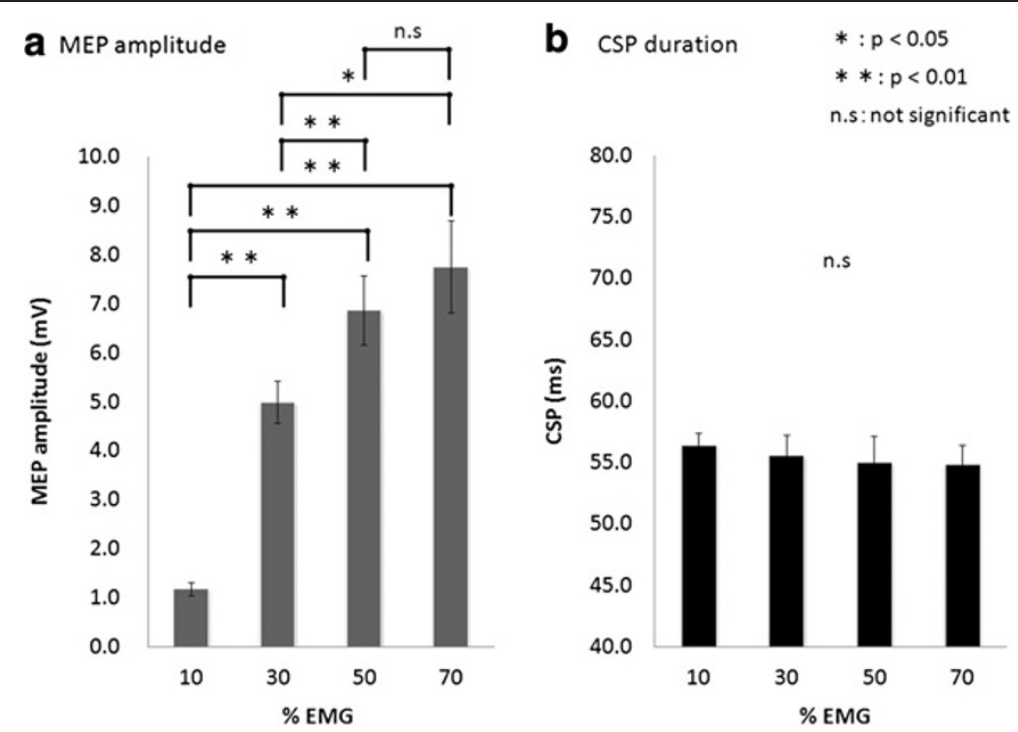

Figure 2 The effects of voluntary muscle contraction on MEP amplitude and CSP duration. (a) The MEP amplitude induced at 30\%, 50\%, and 70\% EMG was significantly larger than that induced at 10\% EMG. The MEP amplitude induced at 50\% and 70\% EMG was significantly larger than that induced at 30\% EMG. (b) CSP duration was not significantly influenced by background contraction.

The MEP amplitude [mean \pm standard error of the mean (SEM)] was $1.17 \pm 0.13 \mathrm{mV}$ at $10 \%$ EMG, $4.98 \pm 0.43 \mathrm{mV}$ at 30\% EMG, $6.86 \pm 0.70 \mathrm{mV}$ at 50\% EMG, and $7.75 \pm 0.94$ $\mathrm{mV}$ at $70 \%$ EMG. The MEP amplitude at 30\%, 50\%, and $70 \%$ EMG was significantly larger than that at $10 \%$ EMG $(\mathrm{p}<0.01)$. Furthermore, the MEP amplitude at $50 \%$ and $70 \%$ EMG was significantly larger than that at 30\% EMG (30\% EMG, $\mathrm{p}<0.01 ; 50 \%$ EMG, $\mathrm{p}<0.05$ ). No significant difference in the MEP amplitude was observed between 50\% and 70\% EMG.

CSP duration [mean \pm standard error of the mean (SEM)] was $56.3 \pm 1.0 \mathrm{~ms}$ at $10 \% \mathrm{EMG}, 55.5 \pm 1.6 \mathrm{~ms}$ at $30 \% \mathrm{EMG}, 55.0 \pm 2.1 \mathrm{~ms}$ at $50 \% \mathrm{EMG}$, and $54.8 \pm 1.6 \mathrm{~ms}$ at $70 \%$ EMG. No significant differences in CSP duration were observed among the muscle contraction levels $\left(\mathrm{F}_{(2.134,21.342)}=0.466, \mathrm{p}>0.05\right.$; Figure $\left.2 \mathrm{~b}\right)$.

\section{Effects of TMS intensity on MEP amplitude and CSP duration (experiment 2)}

Results of one-way ANOVA showed that MEP amplitude changed significantly with increases in TMS intensity $\left(\mathrm{F}_{(2.356,23.564)}=67.687, \mathrm{p}<0.01 ;\right.$ Figure 3a). The MEP amplitude at $10 \%$ EMG was $1.19 \pm 0.13 \mathrm{mV}$ at $100 \%$ AMT, $2.32 \pm 0.33 \mathrm{mV}$ at $110 \%$ AMT, $3.78 \pm 0.50 \mathrm{mV}$ at $120 \%$ AMT, $5.67 \pm 0.77 \mathrm{mV}$ at $130 \%$ AMT, $7.18 \pm 0.66$ $\mathrm{mV}$ at $140 \% \mathrm{AMT}, 7.69 \pm 0.64 \mathrm{mV}$ at $150 \% \mathrm{AMT}$, and $8.28 \pm 0.64 \mathrm{mV}$ at $160 \%$ AMT. No significant difference in the MEP amplitude was observed at high-output stimulation intensities of $>140 \%$ AMT.

Results of one-way ANOVA showed that CSP duration changed significantly with increases in TMS intensity
$\left(\mathrm{F}_{(6,60)}=119.578, \mathrm{p}<0.01\right.$; Figure $\left.3 \mathrm{~b}\right)$. The mean CSP duration was $57.1 \pm 1.0 \mathrm{~ms}$ at $100 \% \mathrm{AMT}, 65.6 \pm 1.6 \mathrm{~ms}$ at $110 \% \mathrm{AMT}, 81.7 \pm 4.2 \mathrm{~ms}$ at $120 \% \mathrm{AMT}, 107.0 \pm 7.1 \mathrm{~ms}$ at $130 \% \mathrm{AMT}, 124.8 \pm 6.6 \mathrm{~ms}$ at $140 \% \mathrm{AMT}, 139.9 \pm 6.0$ $\mathrm{ms}$ at $150 \% \mathrm{AMT}$, and $157.8 \pm 7.0 \mathrm{~ms}$ at $160 \%$ AMT. CSP duration was prolonged with increasing stimulus intensity. CSP duration at $160 \%$ AMT was significantly prolonged compared with those at all other stimulus intensities.

\section{Effects of paired-pulse TMS on MEP amplitude and CSP duration (experiment 3 )}

The mean $( \pm \mathrm{SD})$ conditioning pulse intensity was $32.5 \pm$ $5.8 \%$, and the test pulse intensity was $56.5 \pm 7.5 \%$. Figure 4 shows the representative waveforms of MEP induced by paired-pulse TMS at 10\% EMG. Results of one-way ANOVA showed that MEP amplitude was significantly changed by paired-pulse TMS (rest, $\mathrm{F}_{(2,20)}=18.712$, $\mathrm{p}<0.01 ; 10 \%$ EMG, $\mathrm{F}_{(2,20)}=12.263, \mathrm{p}<0.01 ; 30 \%$ EMG, $\mathrm{F}_{(1.785,17.845)}=21.738, \mathrm{p}<0.01 ; 50 \%$ EMG, $\mathrm{F}_{(1.770,17.698)}=$ $6.788, \mathrm{p}<0.05)$. The mean $( \pm$ SEM) MEP amplitude at rest was $0.90 \pm 0.10 \mathrm{mV}$ (single-pulse TMS), $0.51 \pm 0.08 \mathrm{mV}$ (SICI), and $1.25 \pm 0.18 \mathrm{mV}$ (ICF). The MEP amplitude induced by SICI was significantly smaller than that induced by single-pulse TMS $(\mathrm{p}<0.01)$. Furthermore, the MEP amplitude induced by ICF was significantly larger than that induced by single-pulse TMS $(\mathrm{p}<0.05)$ (Figure $5 \mathrm{a}$ ). The MEP amplitude at 10\% EMG was $7.96 \pm 1.02 \mathrm{mV}$ (single-pulse TMS), $6.33 \pm 0.84 \mathrm{mV}$ (SICI), and $8.95 \pm$ $1.14 \mathrm{mV}$ (ICF) (Figure 5b) and that at 30\% EMG was $10.40 \pm 1.09 \mathrm{mV}$ (single-pulse TMS), $9.53 \pm 0.98 \mathrm{mV}$ (SICI), and $10.90 \pm 1.10 \mathrm{mV}$ (ICF) (Figure 5c). At both 

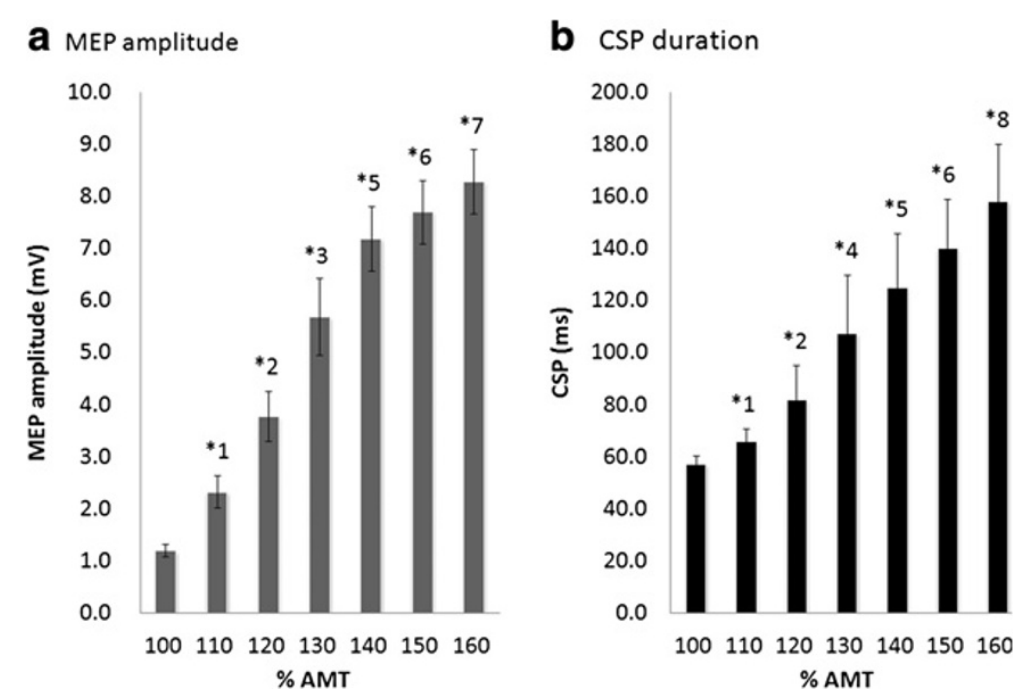

Figure 3 The effects of different stimulation intensities on MEP amplitude and CSP duration at 10\% EMG. (a) The MEP amplitude increased significantly as the stimulus intensity increased from 100\% to 140\% AMT. However, at stimulus intensities $>140 \%$ AMT, no significant differences were observed in the MEP amplitudes. (b) CSP duration increased significantly as the stimulus intensity increased from 100\% to $160 \%$ AMT. *1: 110\% AMT > 100\% AMT ( $p<0.01)$. *2: 120\% AMT > 100\% AMT, $110 \%$ AMT $(p<0.01) .{ }^{*} 3: 130 \%$ AMT > 100\% AMT, 110\% AMT, $120 \%$ AMT $(p<0.01) .{ }^{*} 4: 130 \%$ AMT > 100\% AMT, 110\% AMT ( $\left.p<0.01\right)$. *5: 140\% AMT > 100\% AMT, 110\% AMT, 120\% AMT, 130\% AMT ( $\left.p<0.01\right)$. *6: 150\% AMT > 100\% AMT, 110\% AMT, 120\% AMT, 130\% AMT ( $p<0.01$ ). * $7: 160 \%$ AMT > 100\% AMT, 110\% AMT, 120\% AMT, 130\% AMT (p<0.01). *8: 160\% AMT $>100 \%$ AMT, 110\% AMT, 120\% AMT, 130\% AMT, 140\% AMT, 150\% AMT ( $p<0.01)$.

$10 \%$ and $30 \%$ muscle contraction levels, the MEP amplitude induced by SICI was significantly smaller than that induced by single-pulse TMS and ICF $(\mathrm{p}<0.05)$. Furthermore, the MEP amplitude induced by ICF was significantly larger than that induced by single-pulse TMS at $10 \%$ and $30 \%$ muscle contraction levels ( $\mathrm{p}<$ 0.05). The MEP amplitude at 50\% EMG was $9.67 \pm 0.82$ $\mathrm{mV}$ (single-pulse TMS), $9.06 \pm 0.74 \mathrm{mV}$ (SICI), and $9.73 \pm 0.74 \mathrm{mV}$ (ICF), and the MEP amplitude induced by SICI was significantly smaller than that induced by single-pulse TMS and ICF $(\mathrm{p}<0.05)$. In contrast, no significant differences were observed in the MEP amplitude induced by single-pulse TMS and ICF (Figure 5d).

Results of one-way ANOVA showed that the decrease and increase ratios changed significantly with increases in muscle contractions (decrease ratios, $\mathrm{F}_{(3,30)}=27.744$, $\mathrm{p}<0.01$; increase ratios, $\mathrm{F}_{(1.253,12.530)}=11.571, \mathrm{p}<0.01$; Figure 6). The decrease ratios with SICI were $42.3 \pm$ 6.7\% (rest), $19.2 \pm 4.6 \%$ (10\% EMG), $8.0 \pm 1.5 \%$ (30\% EMG), and $6.7 \pm 1.2 \%$ (50\% EMG). The ratio at rest was significantly higher than that at all muscle contraction levels $(\mathrm{p}<0.05)$, and no significant differences were

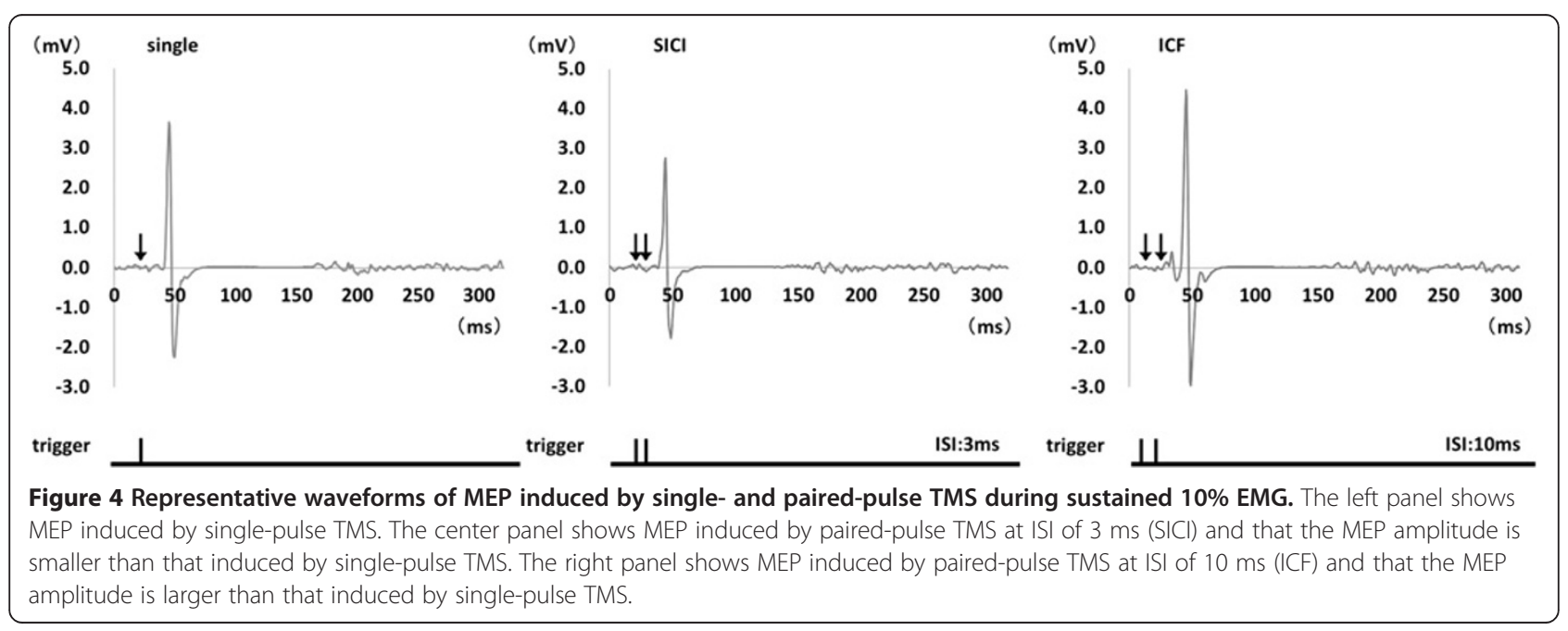




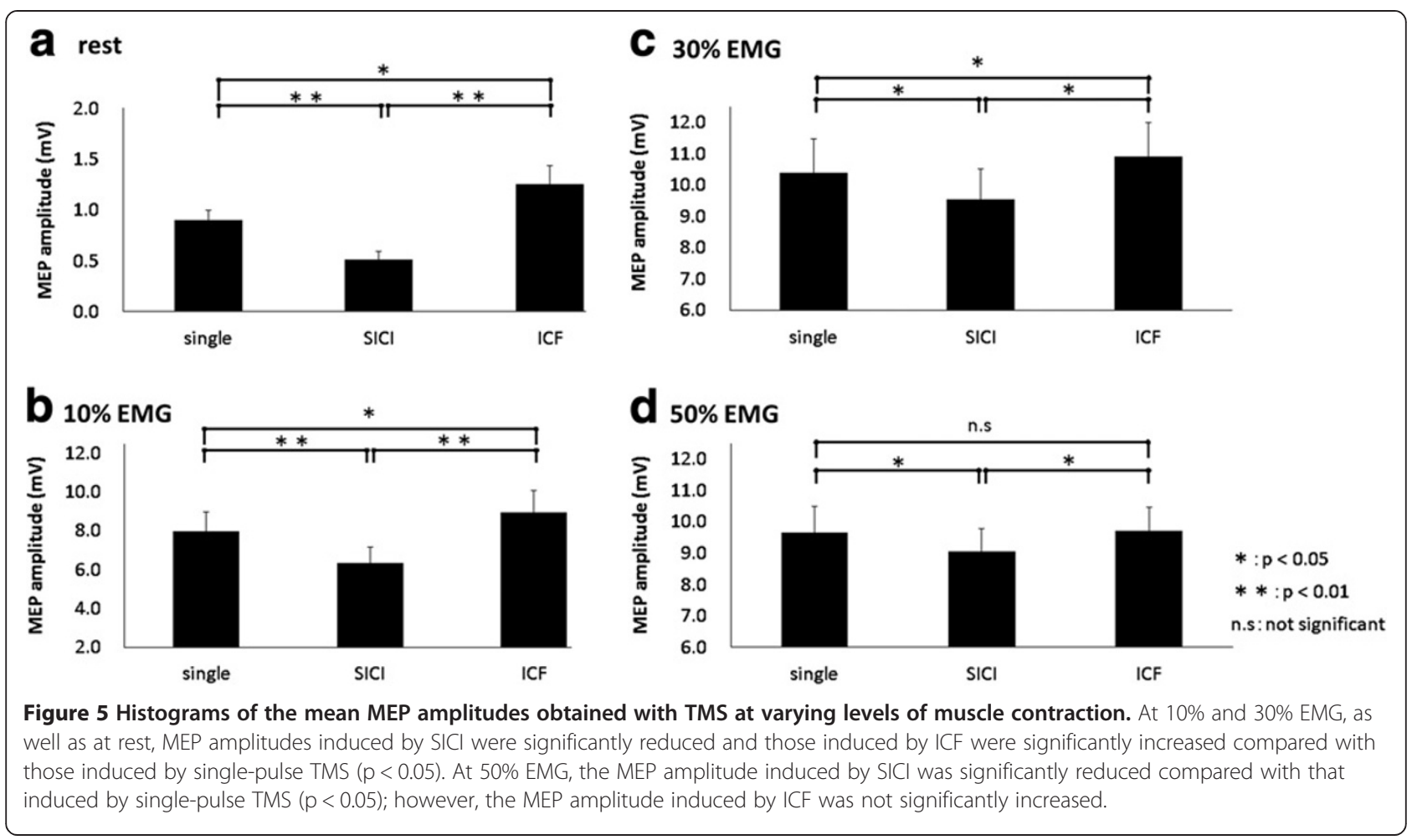

observed among the different muscle contraction levels. The increase ratios with ICF were $41.5 \pm 9.4 \%$ (rest), $12.5 \pm 2.8 \%$ (10\% EMG), $6.1 \pm 1.8 \%$ (30\% EMG), and $4.9 \pm$ $1.2 \%$ (50\% EMG). The ratio at rest was significantly higher than that at $30 \%$ and 50\% EMG $(\mathrm{p}<0.05)$, and no significant differences were observed among the different muscle contraction levels.
Results of one-way ANOVA showed that CSP duration changed significantly with paired-pulse TMS (10\% EMG, $\mathrm{F}_{(1.476,14.756)}=12.734, \mathrm{p}<0.01 ; 30 \%$ EMG, $\mathrm{F}_{(1.988,19.883)}=$ $15.789, \mathrm{p}<0.01 ; 50 \%$ EMG, $\mathrm{F}_{(1.801,18.011)}=24.969, \mathrm{p}<$ 0.01 ; Figure 7). CSP duration at 10\% EMG was $145.5 \pm$ $7.9 \mathrm{~ms}$ (single-pulse TMS), $130.8 \pm 8.6 \mathrm{~ms}$ (SICI), and $145.9 \pm 9.6 \mathrm{~ms}$ (ICF). CSP duration at 30\% EMG was

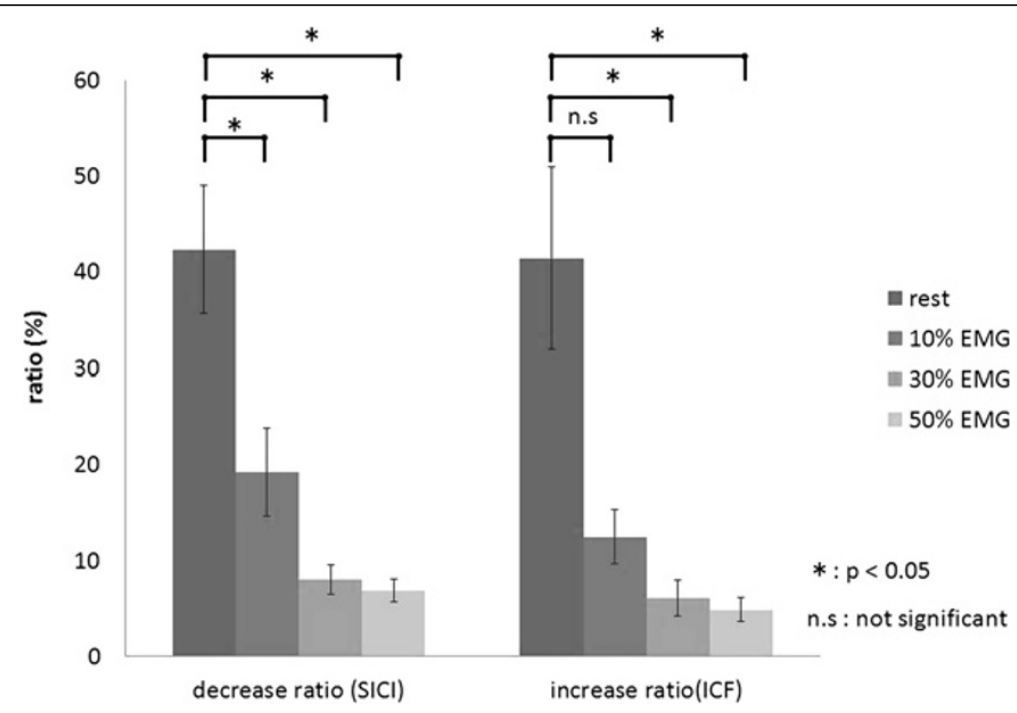

Figure 6 The effects of voluntary muscle contraction on the decrease and increase ratios of MEP amplitudes. Among the decrease ratios, the ratio at rest was significantly higher than those at all muscle contraction levels $(p<0.05)$, and no significant differences were observed at all muscle contraction levels. In contrast, among the increase ratios, the ratio at rest was significantly higher than those at muscle contractions of $30 \%$ and $50 \%$ EMG $(p<0.05)$, and no significant differences were observed at all muscle contraction levels. 


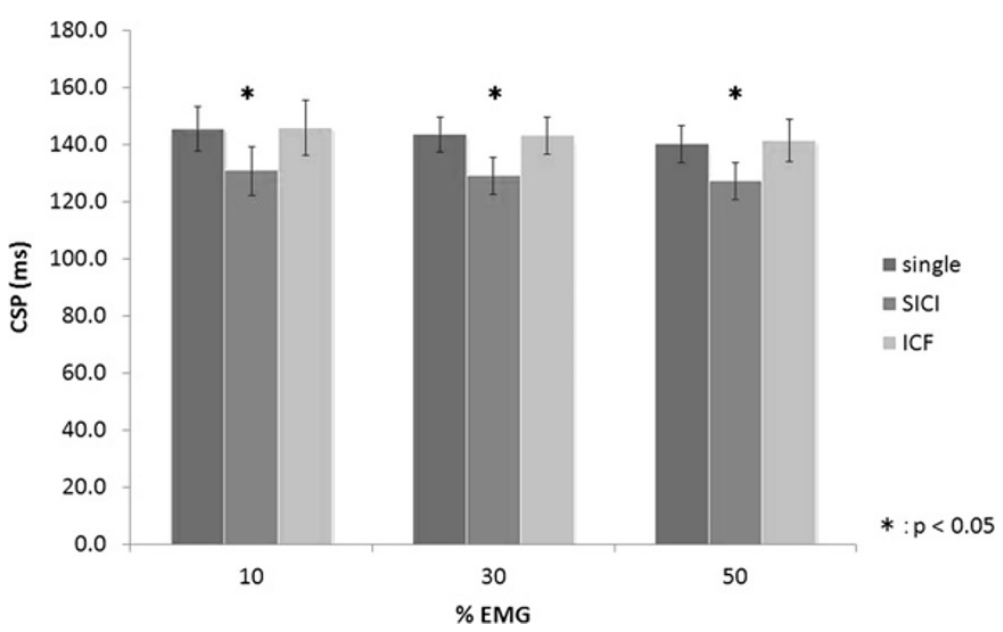

Figure 7 Histograms of the mean CSP durations with TMS at varying muscle contraction levels. At all muscle contraction levels, the duration of CSP after SICI was significantly shorter than that induced by single-pulse TMS and after ICF $(p<0.05)$. No significant difference in CSP duration was observed between single-pulse TMS and ICF.

$143.5 \pm 6.1 \mathrm{~ms}$ (single-pulse TMS), $129.1 \pm 6.5 \mathrm{~ms}$ (SICI), and $143.1 \pm 6.4 \mathrm{~ms}$ (ICF). CSP duration at 50\% EMG was $140.1 \pm 6.5 \mathrm{~ms}$ (single-pulse TMS), $127.3 \pm 6.5 \mathrm{~ms}$ (SICI), and 141.4 $\pm 7.4 \mathrm{~ms}$ (ICF). At all muscle contraction levels, CSP shortened significantly with SICI compared with single-pulse TMS and ICF $(\mathrm{p}<0.05)$. However, no significant differences were observed in CSP duration between single-pulse TMS and ICF.

No significant correlations were observed between MEP amplitude and the duration of CSP induced by single-pulse TMS and after SICI and ICF at 10\%, 30\%, and 50\% EMG (single-pulse TMS, $\mathrm{p}=0.261,0.140$, and 0.345 ; SICI, $\mathrm{p}=0.716,0.234$, and 0.312; ICF, $\mathrm{p}=0.189$, 0.091, and 0.112, respectively; Figure 8).

\section{Discussion}

We confirmed that CSP duration is affected by TMS intensity but not by the muscle contraction level or MEP amplitude. Our new findings were that CSP is shortened with SICI, but it is not altered by ICF, when the TMS intensity is constant. These results indicate that after SICI, CSP duration is affected by the activity of inhibitory intermediate neurons that are activated by the conditioning SICI stimulus.

\section{Effects of muscle contraction on MEP amplitude and CSP duration (experiment 1)}

The MEP amplitude increased with an increase in muscle contraction intensity from $10 \%$ to $50 \%$ EMG in the present study. This result is in agreement with the results of previous reports [7,27,28]. However, we did not observe an increase in MEP amplitude above 50\% EMG despite the increase in MEP amplitude due to an increase in muscle contraction intensity. The most motor units of the first dorsal interosseous (FDI) muscle are recruited below 50\% MVC [29], whereas the maximum recruitment threshold of other large muscles is $95 \%$ or $90 \%$ MVC [30]. We consider that an increase in MEP amplitude in a muscle contraction state above $50 \%$ EMG could not be observed because most of the FDI motor units had already been recruited.

In contrast, CSP duration was always constant and was not influenced by muscle contraction intensity. CSP results from the activity of inhibitory intermediate neurons attached to pyramidal cells of the motor cortex $[4,6]$. Because the stimulation threshold that induces CSP is of a lower intensity than that which induces MEP $[31,32]$, inhibitory intermediate neurons that generate CSP and pyramidal cells involved in generating MEP through stimulation at $100 \%$ AMT can be stimulated [33]. The results of our experiment show that although changes in cortical excitability as a result of muscle contraction have an effect on the excitability of pyramidal cells for MEP generation, they have no effect on the inhibitory intermediate neurons that induce CSP. It has been reported that high MEP amplitude induces a long CSP [6], but the results of experiment 1 support reports that MEP amplitude and CSP fluctuate independently $[4,7,10,11,34-36]$.

\section{Effects of TMS intensity on MEP amplitude and CSP duration (experiment 2)}

We did not observe an increase in MEP above a stimulation intensity of $140 \%$ despite the MEP amplitude increasing with an increase in TMS intensity. Because many motor units are already excited at a stimulation intensity above $140 \%$ AMT, we may not have observed a further increase. Nonetheless, CSP was prolonged with 


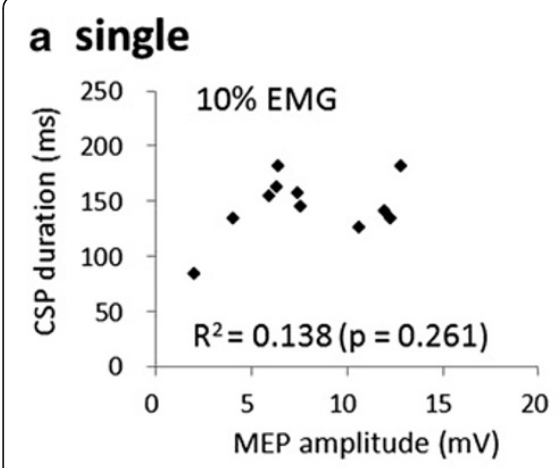

\section{b $\mathrm{SICl}$}

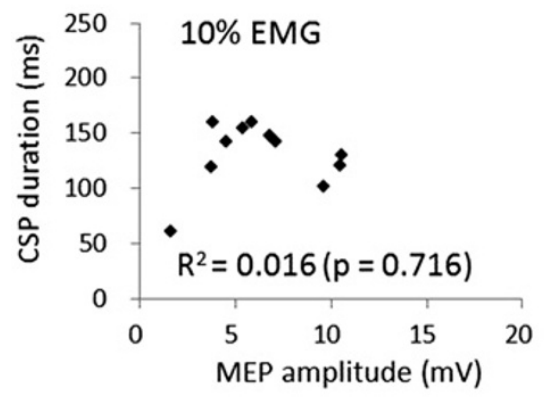

\section{c ICF}

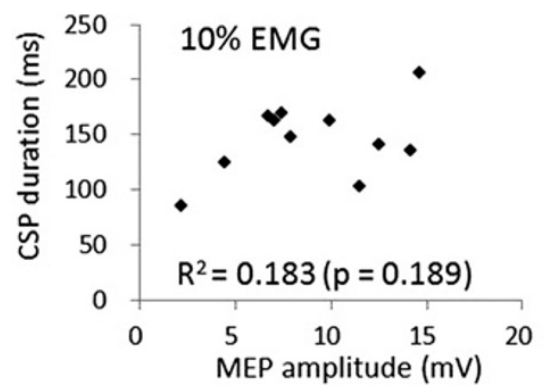

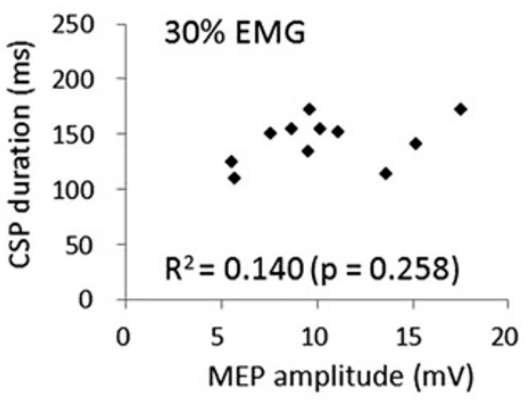
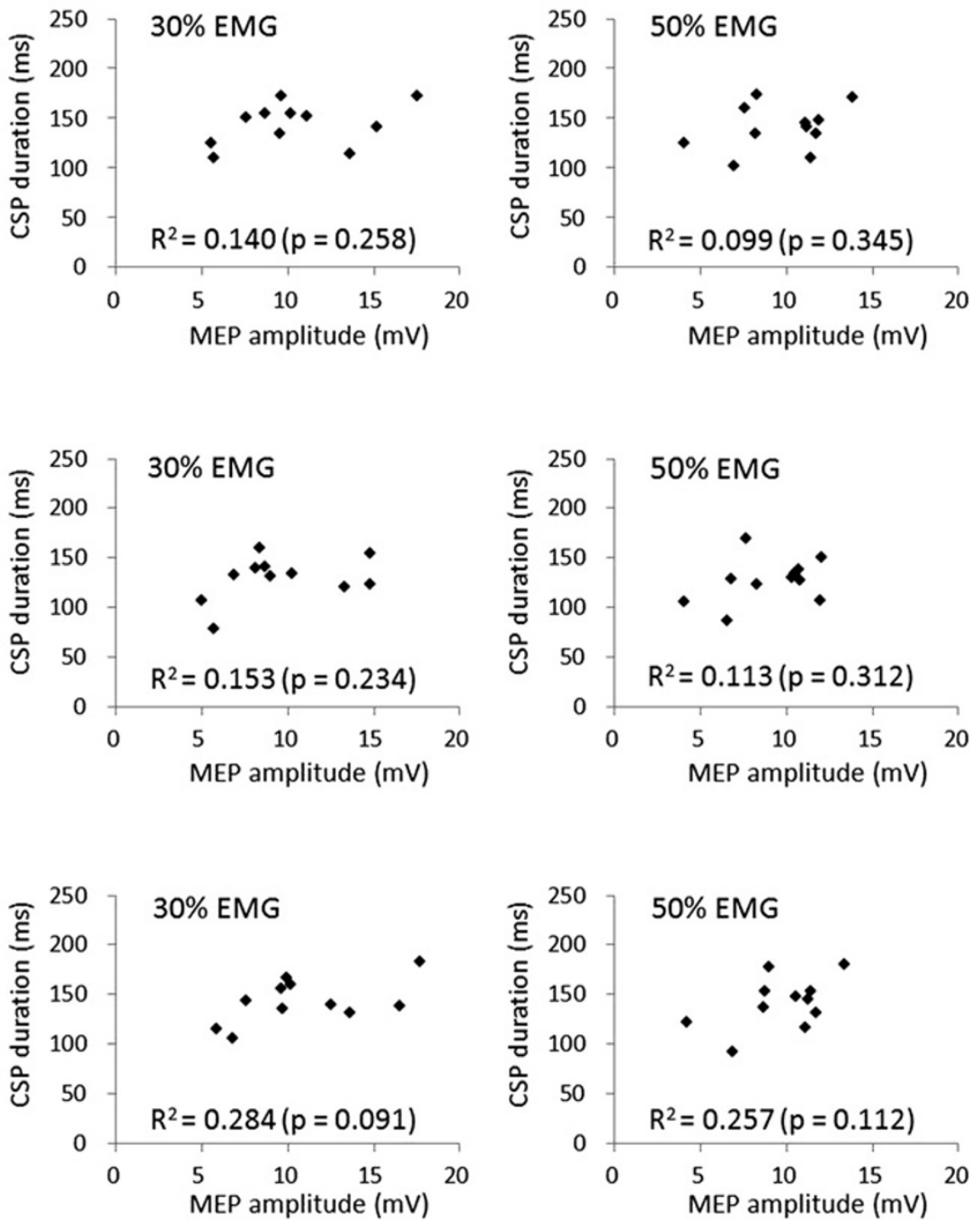

Figure 8 Relationships between MEP and CSP. No significant correlations were observed between MEP amplitude and CSP duration under any of the conditions.

TMS intensity increase from 100\% AMT to 160\% AMT. These results support the reports by Orth et al. [33] and Kimiskidis et al. [37] and are considered to show that CSP is not related to muscle contraction intensity or MEP amplitude, but it varies depending on magnetic stimulation intensity.

Effects of paired-pulse TMS on MEP amplitude and CSP duration (experiment 3)

SICI and ICF were also observed at 10\% and 30\% EMG, similar to SICI and ICF at rest. In contrast, although SICI was observed at 50\% EMG, ICF was not. Ilić TV et al. [19] reported that SICI was observed with paired-pulse stimulation during approximately $5 \%-10 \%$ muscle contractions, whereas ICF was attenuated compared with ICF at rest. In the present experiment, the facilitation effect as a result of ICF was attenuated with increasing muscle contraction intensity from 10\% to 50\% EMG. ICF disappeared at 50\% EMG, but many motor units were already in a mobilized state at 50\% EMG, similar to the results in experiment 1 , which could explain why no ICF occurred.

In contrast, SICI was observed even at an intensity of 50\% EMG, despite the inhibitory effect resulting from SICI attenuation with an increase in muscle contraction intensity. Ortu et al. [25] measured MEP resulting from paired-pulse stimulation at 10\%, 25\%, and 50\% MVC and reported observing SICI at 10\% MVC but not at $25 \%$ or $50 \%$. That study was conducted at an intensity of test stimuli that induced a $1 \mathrm{mV}$ MEP amplitude at each muscle contraction intensity. The present study may 
have produced different results because the magnetic stimulation intensity that induced $1 \mathrm{mV}$ at rest was used at all muscle contraction levels.

CSP was significantly shortened with SICI compared with single-pulse TMS and ICF at all muscle contraction intensities, whereas no significant differences in CSP duration were observed between ICF and single-pulse TMS. A few studies have reported the modulation of CSP by paired-pulse TMS [22-24], but the results have not been consistent. In previous studies, GABAA receptors were reported to be involved in the SICI mechanism $[14,17,19,20]$. CSP is reported to occur when pyramidal cells in the cortex that sustain muscle contractions are suppressed [25]. However, GABAA-mediated inhibitory interneurons were excited because of the conditioning pulse of SICI and because the activity of inhibitory intermediate neurons that cause CSP and the proliferation of pyramidal cells involved in MEP were suppressed, CSP was shortened. There are many uncertainties about the action mechanism of ICF. Di Lazzaro et al. [21] reported that ICF is a change caused by the excitability of the spinal cord, whereas Nakamura et al. [15] suggested the possibility of facilitation within the cortex. Our present experiment did not clarify whether the influences of the intracortical or subcortical network cause ICF. However, under conditions in which ISI of paired-pulse stimulation was $10 \mathrm{~ms}$, it was clear that this did not change CSP, although MEP was increased.

\section{Conclusions}

This study investigated the effects of muscle contractions and stimulus intensities on MEP and CSP induced by single-pulse TMS and the effects of SICI and ICF on CSP duration. Our major results were that CSP is affected by TMS intensity but not by the muscle contraction level or MEP amplitude. In addition, although TMS intensities were constant, CSP was significantly shortened with SICI compared with single-pulse TMS and ICF. These results indicated that after SICI, CSP is affected by the activity of inhibitory intermediate neurons that were activated by the conditioning SICI stimulus.

\section{Methods}

\section{Subjects}

Fifteen healthy, right-handed, male volunteers (age, 2143 years; mean \pm SD, $25.1 \pm 5.1$ years) participated in this study. All subjects gave their written informed consent. This study was approved by the ethics committee at the Niigata University of Health and Welfare, Niigata, Japan (17279-111012).

\section{EMG recording}

The subjects were seated comfortably in a chair. EMGs were recorded from the right FDI muscle using a silver/ silver-chloride electrode in a belly tendon montage. EMG signals were amplified $(\times 1000)$ by an amplifier (A-DL-720•140; 4 Assist, Tokyo, Japan) and digitized at $2 \mathrm{kHz}$ using a A/D converter (PowerLab 8/30; AD Instruments, $\mathrm{CO}$, USA). The subjects performed right index finger abduction, and MVC and maximum EMG values of FDI were measured (100\% EMG). The EMG signals were rectified and smoothed with a 501-point smoothing. The muscle contraction level was controlled by displaying a constantly updated bar graph showing the smoothed EMG amplitude (expressed as a percentage of maximal contraction). These monitors also allowed us to confirm the state of muscle contraction. The subjects were instructed to contract and sustain the targeted levels at 0 (rest), 10, 30, 50, and $70 \%$ EMG.

\section{TMS}

Monophasic pulse TMS was delivered with a figureof-eight-shaped coil (diameter, $95 \mathrm{~mm}$ ) connected to Magstim 200 (Magstim, Dyfed, UK). The coil was held with the handle pointing backward and laterally at approximately $45^{\circ}$ to the sagittal plane. The optimal spot to elicit MEP in the right FDI was carefully determined in each subject, and the optimal coil position to evoke a stable MEP was marked on the cap worn by the subject. AMT was obtained during a slight isometric contraction (approximately 5\% MVC) and was defined as the lowest stimulus intensity able to induce MEP with greater than $100 \mu \mathrm{V}$ peak-to-peak amplitude in FDI in at least 5 of 10 consecutive trials [14]. The $1 \mathrm{mV}$ TMS intensity was defined as the lowest stimulus intensity able to induce

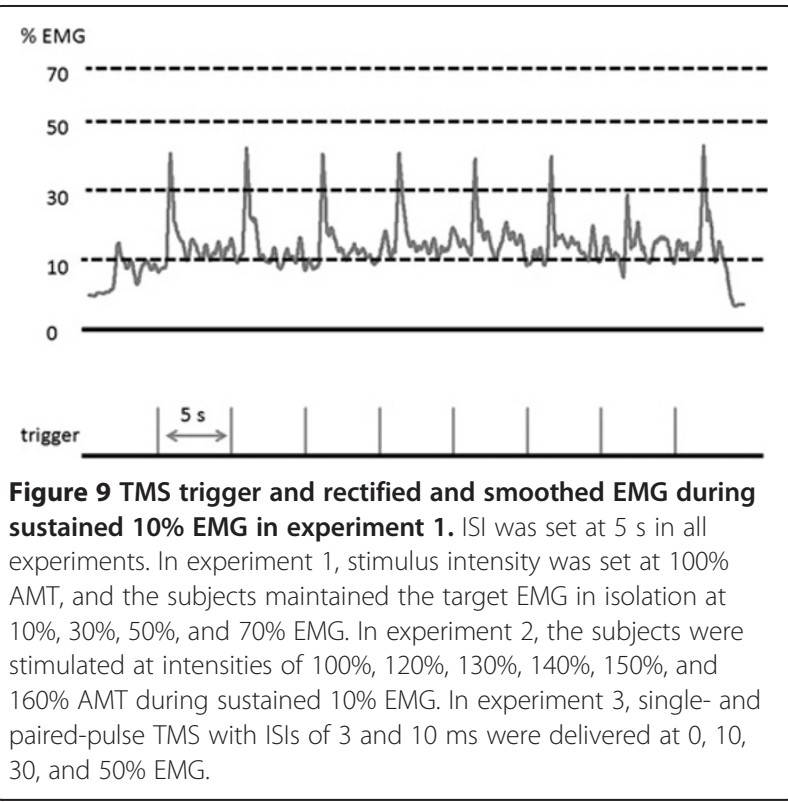


MEP with greater than $1 \mathrm{mV}$ peak-to-peak amplitude in the relaxed FDI in at least 5 of 10 consecutive trials [25].

\section{Experiment 1 (effects of muscle contraction on MEP amplitude and CSP duration)}

The effect of muscle contraction strength on MEP and CSP induced by single-pulse TMS was investigated in 11 subjects (age, $23.3 \pm 2.2$ years). The subjects sustained the target EMG in isolation at 10\%, 30\%, 50\%, and 70\% EMG for 40 s. TMS intensity was 100\% AMT, and 8 stimuli were applied at $0.2 \mathrm{~Hz}$. A representative TMS trigger and rectified and smoothed EMG during sustained 10\% EMG in experiment 1 are shown in Figure 9.

\section{Experiment 2 (effects of TMS intensity on MEP amplitude and CSP duration)}

We investigated the effect of stimulus intensity on MEP and CSP in the same 11 subjects as in experiment 1. Right index finger abduction was performed in the same manner as in experiment 1. The muscle contraction level was 10\% EMG, and TMS intensity was set at 100\%, $110 \%, 120 \%, 130 \%, 140 \%, 150 \%$, and $160 \%$ AMT with $0.2 \mathrm{~Hz}$ frequency. Muscle contraction was sustained for $40 \mathrm{~s}$, and eight stimuli were applied at each TMS intensity level.

\section{Experiment 3 (effects of paired-pulse TMS on MEP amplitude and CSP duration)}

The effects of SICI and ICF induced by paired-pulse TMS on MEP amplitude and CSP duration were investigated in 11 subjects (age, 27.1 \pm 8.2 years, including seven of the same subjects as in experiment 1 ). The stimulus intensities used for paired-pulse TMS were $80 \%$ AMT as a conditioning pulse and $1 \mathrm{mV}$ TMS intensity as the test pulse. These conditioning and test intensities were the same for all muscle contraction levels, because CSP duration might be influenced by TMS intensity [33]. ISI of the paired-pulse TMS was $3 \mathrm{~ms}$ (SICI) and $10 \mathrm{~ms}$ (ICF). SICI and ICF were recorded using three stimulations, including single-pulse TMS, at rest, and three contractions (10\%, 30\%, and 50\% EMG). Pairedpulse TMS of each ISI and single-pulse TMS were randomly presented among a total of 24 stimuli and were applied at $0.2 \mathrm{~Hz}$.

\section{Data analysis}

MEP amplitudes were calculated from peak-to-peak amplitudes, except for the amplitude of the maximum and minimum MEP amplitude of 8 waves. CSP duration was defined as the time from TMS onset to the time of reappearance of EMG amplitude that was more than 3 -fold the SD of the background EMG noise at rest, and the average values were calculated for all CSP durations. The decrease and increase ratios with SICI and ICF, respectively, [absolute (conditioned MEP - unconditioned MEP)/unconditioned MEP $\times 100$ ) were calculated at each muscle contraction level.

Statistical analysis was performed using PASW statistics 18 software (IBM SPSS, Armonk, NY, USA). All data, including MEP amplitudes and CSP durations, were statistically analyzed by one-way repeated measures ANOVA with muscle contractions, stimulus intensities, and ISI as the within-subject factors. The sphericity of data was analyzed by Mauchly's test, and Greenhouse-Geissercorrected significance values were used when sphericity was lacking. Post hoc analysis was performed with Bonferroni's methods for multiple comparisons to avoid type I errors. The correlations between MEP amplitude and the duration of CSP induced by single-pulse TMS and after SICI and ICF at each muscle contraction level were assessed by Pearson's correlation analysis. Differences were considered significant at $\mathrm{p}<0.05$ for all analyses.

\section{Abbreviations}

CSP: Cortical silent period; TMS: Transcranial magnetic stimulation; MEP: Motor evoked potential; SICI: Short-interval intracortical inhibition; ICF: Intracortical facilitation; AMT: Active motor threshold;

EMG: Electromyogram; SD: Standard deviation; SEM: Standard error of the mean; ISI: Interstimulus intervals; MVC: Maximum voluntary contraction; ANOVA: Analysis of variance; FDI: First dorsal interosseous.

\section{Competing interests}

The authors declare no competing interests.

\section{Authors' contributions}

SK conceived of the study, designed the experimental paradigm, performed the statistical analysis, and contributed to the discussion and preparation of the manuscript. $\mathrm{HO}$ conceived of the study, designed the experimental paradigm, performed the statistical analysis, and contributed to the discussion and preparation of the manuscript. KS conceived of the study, designed the experimental paradigm, performed the statistical analysis, and contributed to the discussion and preparation of the manuscript. HK conceived of the study, designed the experimental paradigm, and contributed to the discussion of the manuscript. MS performed the statistical analysis and contributed to the discussion and preparation of the manuscript. HT conceived of the study, designed the experimental paradigm, and contributed to the discussion of the manuscript. All authors read and approved the final manuscript.

\section{Acknowledgments}

This work was supported by a Grant-in-Aid for Advanced Research from Niigata University of Health and Welfare (2012), a Grant-in-Aid from the President of Niigata University of Health and Welfare (2012), and Scientific Research of Graduate Students of Niigata University of Health and Welfare (2012).

\section{Received: 11 November 2012 Accepted: 27 March 2013}

Published: 2 April 2013

\section{References}

1. Brasil-Neto JP, Cammarota A, Valls-Sole J, Pascual-Leone A, Hallett M, Cohen LG: Role of intracortical mechanisms in the late part of the silent period to transcranial stimulation of the human motor cortex. Acta Neurol Scand 1995, 92(5):383-386.

2. Chen R, Lozano AM, Ashby P: Mechanism of the silent period following transcranial magnetic stimulation. Evidence from epidural recordings. Exp Brain Res 1999, 128(4):539-542. 
3. Tergau F, Wanschura V, Canelo M, Wischer S, Wassermann EM, Ziemann U, Paulus W: Complete suppression of voluntary motor drive during the silent period after transcranial magnetic stimulation. Exp Brain Res 1999, 124(4):447-454

4. Wilson SA, Lockwood RJ, Thickbroom GW, Mastaglia FL: The muscle silent period following transcranial magnetic cortical stimulation. J Neuro/ Sci 1993, 114(2):216-222.

5. Ziemann U, Netz J, Szelenyi A, Homberg V: Spinal and supraspinal mechanisms contribute to the silent period in the contracting soleus muscle after transcranial magnetic stimulation of human motor cortex. Neurosci Lett 1993, 156(1-2):167-171.

6. Inghilleri M, Berardelli A, Cruccu G, Manfredi M: Silent period evoked by transcranial stimulation of the human cortex and cervicomedullary junction. J Physiol 1993, 466:521-534.

7. Taylor JL, Allen GM, Butler JE, Gandevia SC: Effect of contraction strength on responses in biceps brachii and adductor pollicis to transcranial magnetic stimulation. Exp Brain Res 1997, 117(3):472-478.

8. Tazoe T, Sakamoto M, Nakajima T, Endoh T, Komiyama T: Effects of remote muscle contraction on transcranial magnetic stimulation-induced motor evoked potentials and silent periods in humans. Clin Neurophysio/ 2007, 118(6):1204-1212.

9. Tinazzi M, Farina S, Tamburin S, Facchini S, Fiaschi A, Restivo D, Berardelli A: Task-dependent modulation of excitatory and inhibitory functions within the human primary motor cortex. Exp Brain Res 2003, 150(2):222-229.

10. Wu L, Goto Y, Taniwaki T, Kinukawa N, Tobimatsu S: Different patterns of excitation and inhibition of the small hand and forearm muscles from magnetic brain stimulation in humans. Clin Neurophysiol 2002, 113(8):1286-1294.

11. Gilio F, Rizzo V, Siebner HR, Rothwell JC: Effects on the right motor handarea excitability produced by low-frequency rTMS over human contralateral homologous cortex. J Physiol 2003, 551(Pt 2):563-573.

12. Kujirai T, Caramia MD, Rothwell JC, Day BL, Thompson PD, Ferbert A, Wroe S, Asselman P, Marsden CD: Corticocortical inhibition in human motor cortex. J Physiol 1993, 471:501-519.

13. Ridding MC, Taylor JL, Rothwell JC: The effect of voluntary contraction on cortico-cortical inhibition in human motor cortex. J Physiol 1995, 487(Pt 2):541-548.

14. Ziemann U, Rothwell JC, Ridding MC: Interaction between intracortical inhibition and facilitation in human motor cortex. J Physiol 1996, 496(Pt 3):873-881.

15. Nakamura H, Kitagawa H, Kawaguchi $Y$, Tsuji H: Intracortical facilitation and inhibition after transcranial magnetic stimulation in conscious humans. J Physiol 1997, 498(Pt 3):817-823.

16. Di Lazzaro $V$, Restuccia D, Oliviero A, Profice $P$, Ferrara L, Insola A, Mazzone $P$, Tonali $P$, Rothwell JC: Magnetic transcranial stimulation at intensities below active motor threshold activates intracortical inhibitory circuits. Exp Brain Res 1998, 119(2):265-268.

17. Di Lazzaro V, Oliviero A, Meglio M, Cioni B, Tamburrini G, Tonali P, Rothwell $J C$ : Direct demonstration of the effect of lorazepam on the excitability of the human motor cortex. Clin Neurophysiol 2000, 111(5):794-799.

18. Hanajima R, Ugawa Y, Terao Y, Sakai K, Furubayashi T, Machii K, Kanazawa I: Paired-pulse magnetic stimulation of the human motor cortex: differences among I waves. J Physiol 1998, 509(Pt 2):607-618.

19. Ilic TV, Meintzschel F, Cleff U, Ruge D, Kessler KR, Ziemann U: Short-interval paired-pulse inhibition and facilitation of human motor cortex: the dimension of stimulus intensity. J Physio/ 2002, 545(1):153-167.

20. Korchounov A, Ilic TV, Schwinge T, Ziemann U: Modification of motor cortical excitability by an acetylcholinesterase inhibitor. Exp Brain Res 2005, 164(3):399-405

21. Di Lazzaro V, Pilato F, Oliviero A, Dileone M, Saturno E, Mazzone P, Insola A, Profice $P$, Ranieri F, Capone F, et al: Origin of facilitation of motor-evoked potentials after paired magnetic stimulation: direct recording of epidural activity in conscious humans. J Neurophysio/ 2006, 96(4):1765-1771.

22. Kang SY, Shin HW, Sohn YH: Different modulation of the cortical silent period by two phases of short interval intracortical inhibition. Yonsei Med J 2007, 48(5):795-801.

23. Shimizu T, Oliveri M, Filippi MM, Palmieri MG, Pasqualetti P, Rossini PM: Effect of paired transcranial magnetic stimulation on the cortical silent period. Brain Res 1999, 834(1-2):74-82.
24. Trompetto C, Buccolieri A, Marinelli L, Abbruzzese G: Differential modulation of motor evoked potential and silent period by activation of intracortical inhibitory circuits. Clin Neurophysiol 2001, 112(10):1822-1827.

25. Ortu E, Deriu F, Suppa A, Tolu E, Rothwell JC: Effects of volitional contraction on intracortical inhibition and facilitation in the human motor cortex. J Physiol 2008, 586(Pt 21):5147-5159.

26. Roshan L, Paradiso GO, Chen R: Two phases of short-interval intracortical inhibition. Exp Brain Res 2003, 151(3):330-337.

27. Sacco P, Thickbroom GW, Thompson ML, Mastaglia FL: Changes in corticomotor excitation and inhibition during prolonged submaximal muscle contractions. Muscle Nerve 1997, 20(9):1158-1166.

28. Ugawa Y, Terao Y, Hanajima R, Sakai K, Kanazawa I: Facilitatory effect of tonic voluntary contraction on responses to motor cortex stimulation Electroencephalogr Clin Neurophysiol 1995, 97(6):451-454.

29. Duchateau J, Hainaut K: Effects of immobilization on contractile properties, recruitment and firing rates of human motor units. J Physiol 1990, 422:55-65.

30. De Luca CJ, Hostage EC: Relationship between firing rate and recruitment threshold of motoneurons in voluntary isometric contractions. J Neurophysiol 2010, 104(2):1034-1046.

31. Chen R, Tam A, Butefisch C, Corwell B, Ziemann U, Rothwell JC, Cohen LG: Intracortical inhibition and facilitation in different representations of the human motor cortex. J Neurophysio/ 1998, 80(6):2870-2881.

32. Davey NJ, Romaiguere P, Maskill DW, Ellaway PH: Suppression of voluntary motor activity revealed using transcranial magnetic stimulation of the motor cortex in man. J Physio/ 1994, 477(Pt 2):223-235.

33. Orth M, Rothwell JC: The cortical silent period: intrinsic variability and relation to the waveform of the transcranial magnetic stimulation pulse. Clin Neurophysiol 2004, 115(5):1076-1082.

34. Cantello R, Gianelli M, Civardi C, Mutani R: Magnetic brain stimulation: the silent period after the motor evoked potential. Neurology 1992, 42(10):1951-1959.

35. Uozumi T, Ito $Y$, Tsuji S, Murai $Y$ : Inhibitory period following motor potentials evoked by magnetic cortical stimulation. Electroencephalogr Clin Neurophysio/ 1992, 85(4):273-279.

36. Ziemann U: TMS and drugs. Clin Neurophysiol 2004, 115(8):1717-1729.

37. Kimiskidis VK, Papagiannopoulos S, Sotirakoglou K, Kazis DA, Kazis A, Mills KR: Silent period to transcranial magnetic stimulation: construction and properties of stimulus-response curves in healthy volunteers. Exp Brain Res 2005, 163(1):21-31.

doi:10.1186/1471-2202-14-43

Cite this article as: Kojima et al.: Modulation of the cortical silent period elicited by single- and paired-pulse transcranial magnetic stimulation. BMC Neuroscience 2013 14:43.

\section{Submit your next manuscript to BioMed Central and take full advantage of:}

- Convenient online submission

- Thorough peer review

- No space constraints or color figure charges

- Immediate publication on acceptance

- Inclusion in PubMed, CAS, Scopus and Google Scholar

- Research which is freely available for redistribution 